\title{
Dissipative effects on the sustainment of a magnetorotational dynamo in Keplerian shear flow
}

\author{
A. Riols ${ }^{1,2}$, F. Rincon ${ }^{1,2}$, C. Cossu $^{3}$, G. Lesur ${ }^{4,5}$, G. I. Ogilvie ${ }^{6}$, and P. -Y. Longaretti ${ }^{4,5}$ \\ 1 Université de Toulouse, UPS-OMP, IRAP Toulouse, France \\ e-mail: antoine.riols@irap.omp.eu \\ 2 CNRS, IRAP, 14 avenue Edouard Belin, 31400 Toulouse, France \\ 3 CNRS, Institut de Mécanique des Fluides de Toulouse (IMFT), Allée du Professeur Camille Soula, 31400 Toulouse, France \\ ${ }^{4}$ Univ. Grenoble Alpes, IPAG, 38000 Grenoble, France \\ 5 CNRS, IPAG, 38000 Grenoble, France \\ ${ }^{6}$ Department of Applied Mathematics and Theoretical Physics, University of Cambridge, Centre for Mathematical Sciences, \\ Wilberforce Road, Cambridge CB3 0WA, UK
}

Received 2 June 2014 / Accepted 4 October 2014

\begin{abstract}
The magnetorotational (MRI) dynamo has long been considered one of the possible drivers of turbulent angular momentum transport in astrophysical accretion disks. However, various numerical results suggest that this dynamo may be difficult to excite in the astrophysically relevant regime of magnetic Prandtl number $(\mathrm{Pm})$ significantly smaller than unity, for reasons currently not well understood. The aim of this article is to present the first results of an ongoing numerical investigation of the role of both linear and nonlinear dissipative effects in this problem. Combining a parametric exploration and an energy analysis of incompressible nonlinear MRI dynamo cycles representative of the transitional dynamics in large aspect ratio shearing boxes, we find that turbulent magnetic diffusion makes the excitation and sustainment of this dynamo at moderate magnetic Reynolds number $(\mathrm{Rm})$ increasingly difficult for decreasing Pm. This results in an increase in the critical Rm of the dynamo for increasing kinematic Reynolds number (Re), in agreement with earlier numerical results. Given its very generic nature, we argue that turbulent magnetic diffusion could be an important determinant of MRI dynamo excitation in disks, and may also limit the efficiency of angular momentum transport by MRI turbulence in low Pm regimes.
\end{abstract}

Key words. accretion, accretion disks - dynamo - instabilities - magnetohydrodynamics (MHD) - turbulence

\section{Introduction}

Magnetorotational instability (MRI) occurs in differentially rotating flows whose angular velocity decreases with distance to the rotation axis (Velikhov 1959; Chandrasekhar 1960; Balbus \& Hawley 1991) and is the most commonly invoked excitation mechanism of angular momentum-transporting turbulence in accretion disks (Balbus \& Hawley 1998). In a uniform magnetic field $\boldsymbol{B}$, the MRI amplifies arbitrarily small perturbations exponentially and breaks down nonlinearly into MHD turbulence (e.g. Hawley et al. 1995). The transport efficiency of MRI turbulence continues to be debated, and may be reduced in the astrophysically relevant regime of low magnetic Prandtl number $(\mathrm{Pm})$, the ratio between kinematic viscosity and magnetic diffusivity (Lesur \& Longaretti 2007; Balbus \& Henri 2008).

Another question in this context is that of the origin of the MRI-supporting magnetic field. In some cases, this field may be generated by an internal disk dynamo process which could bootstrap MHD turbulence in the disk independently of its magnetic environment (Balbus \& Hawley 1998; Donati et al. 2005). Early simulations by Hawley et al. (1996) in the so-called zero net flux configuration appropriate to this problem showed that such a dynamo is indeed possible and is intimately coupled to the MRI (see also Brandenburg et al. 1995), but its viability in disks has since been questioned by numerical studies suggesting that it may be impossible to excite at low Pm (Fromang et al. 2007), although the physical reasons for this are not yet clear (Bodo et al. 2011; Käpylä \& Korpi 2011; Oishi \& Mac Low 2011; Simon et al. 2011).

The aim of this article is to seek a physical explanation for this behaviour by exploiting recently discovered dynamical properties of this subcritical dynamo mechanism (Rincon et al. 2007, 2008; Lesur \& Ogilvie 2008), whose principles are otherwise rather simple: starting from a zero net-flux axisymmetric weak poloidal field, a larger toroidal field is generated through the $\Omega$ effect. This field is MRI-unstable to non-axisymmetric MHD perturbations, whose growth results in a nonlinear electromotive force (EMF) that sustains (and can also reverse) the axisymmetric field. Recent work suggests that three-dimensional cyclic nonlinear solutions provide the first germs of excitation of the dynamo in shearing box simulations (Herault et al. 2011; Riols et al. 2013) and possibly form the backbone of the ensuing self-sustained MHD turbulence. Parametric studies of cycles representative of the transitional dynamics, complemented with an analysis of their energetics, may therefore prove useful to understand how dissipative effects affect the dynamo transition as a whole. Here, we present the first results of an ongoing numerical investigation of this kind. We focus on the simpler case of incompressible dynamics in large aspect ratio shearing boxes, which includes most of the fundamental physical complexity of 
the problem, except for stratification and boundary effects. An exhaustive study of different configurations will be presented in a subsequent paper.

The equations and numerical framework used in this article are presented in Sect. 2. In Sect. 3, we study the characteristics of the transition in elongated shearing boxes using generic incompressible numerical simulations, in order to check if the results of Fromang et al. (2007) on the Pm-dependence of the transition extend to such configurations. We also investigate whether cycles still provide the first germs of MRI dynamo chaos at kinematic Reynolds number ( $\mathrm{Re}$ ) larger than studied by Riols et al. (2013). In Sect. 4, we compute the existence boundaries of several cycles in the magnetic versus kinematic Reynolds number parameter plane and analyse their energy budget to identify physical effects affecting the dynamics in the vicinity of $\mathrm{Pm} \sim 1$. Additional numerical experiments aiming at investigating the conditions of excitation of the dynamo, and why it appears harder to excite at low Pm, are presented in Sect. 5. A short discussion concludes the paper.

\section{Equations and numerical framework}

\subsection{Model}

The equations and numerical framework are the same as in the work of Herault et al. (2011) and Riols et al. (2013) and have already been described in detail in these papers. We use the cartesian local shearing sheet description of differentially rotating flows (Goldreich \& Lynden-Bell 1965), whereby the axisymmetric differential rotation is approximated locally by a linear shear flow $\boldsymbol{U}_{x}=-S x \boldsymbol{e}_{y}$, and a uniform rotation rate $\boldsymbol{\Omega}=\Omega \boldsymbol{e}_{z}$, with $\Omega=(2 / 3) S$ for a Keplerian equilibrium. Here $(x, y, z)$ are respectively the shearwise, streamwise and spanwise directions, corresponding to the radial, azimuthal and vertical directions in accretion disks. We refer to the $(x, z)$ projection of vector fields as their poloidal component and to the $y$ direction as their toroidal component. Stratification and compressibility effects are ignored for simplicity. The evolution of the threedimensional velocity field perturbations $\boldsymbol{u}$ and magnetic field $\boldsymbol{B}$ is governed by the three-dimensional incompressible, dissipative MHD equations:

$$
\frac{\partial \boldsymbol{u}}{\partial t}-S x \frac{\partial \boldsymbol{u}}{\partial y}+\boldsymbol{u} \cdot \boldsymbol{\nabla} \boldsymbol{u}=-2 \boldsymbol{\Omega} \times \boldsymbol{u}+S u_{x} \boldsymbol{e}_{y}-\nabla \Pi+\boldsymbol{B} \cdot \boldsymbol{\nabla} \boldsymbol{B}+v \boldsymbol{\Delta} \boldsymbol{u},
$$

$\frac{\partial \boldsymbol{B}}{\partial t}-S x \frac{\partial \boldsymbol{B}}{\partial y}=-S B_{x} \boldsymbol{e}_{y}+\nabla \times(\boldsymbol{u} \times \boldsymbol{B})+\eta \boldsymbol{\Delta} \boldsymbol{B}$,

$\nabla \cdot \boldsymbol{u}=0, \quad \nabla \cdot \boldsymbol{B}=0$.

The kinematic and magnetic Reynolds numbers are defined by $\operatorname{Re}=S L^{2} / v$ and $\operatorname{Rm}=S L^{2} / \eta$, where $v$ and $\eta$ are the constant kinematic viscosity and magnetic diffusivity, $L$ is a typical scale of the spatial domain and time is measured with respect to $S^{-1}$. The magnetic Prandtl number is $\mathrm{Pm}=v / \eta=\mathrm{Rm} / \mathrm{Re}$. $\Pi$ is the total of fluid plus magnetic pressure divided by the uniform density. $\boldsymbol{B}$ is expressed in terms of an alfvénic velocity. Both $\boldsymbol{u}$ and $\boldsymbol{B}$ are measured in units of $S L$.

\subsection{Numerical methods}

We use the SNOOPY code (Lesur \& Longaretti 2007) to perform direct numerical simulations (DNS) of Eqs. (1)-(3). This code provides a spectral implementation of the so-called numerical shearing box model of the shearing sheet, in a finite domain of size $\left(L_{x}, L_{y}, L_{z}\right)$, at numerical resolution $\left(N_{x}, N_{y}, N_{z}\right)$. The $x$ and $y$ directions are taken as periodic while shear-periodicity is imposed in $x$. A discrete spectral basis of shearing waves with constant $k_{y}$ and $k_{z}$ wavenumbers and constant shearwise Lagrangian wavenumber $k_{x_{0}}$ is used to represent the fields in the sheared Lagrangian frame. The shearing of nonaxisymmetric perturbations in this model $\left(k_{y} \neq 0\right)$ is described using time-dependent Eulerian shearwise wavenumbers, $k_{x}(t)=k_{x_{0}}+S k_{y} t$. Shearing waves are "leading" when $k_{x} k_{y}<0$ and "trailing" when $k_{x} k_{y}>0$.

Nonlinear periodic solutions are computed with the NewtonKrylov solver PEANUTS interfaced to SNOOPY, and followed in parameter space using arclength continuation. Almost all the results presented in the paper are for a maximum resolution of $(48,48,72)$, ensuring convergence for all parameters considered, except for some of the results of Sect. 3.1 which required a higher resolution.

\subsection{Symmetries and aspect ratio choice}

The dynamics in the transitional regimes typical of simulations displaying recurrent dynamics ( $\mathrm{Re}$ and $\mathrm{Rm}$ of a few hundreds to thousands) is already quite complex and clearly involves a large number of cyclic solutions. Given the current state of understanding of the dependence of the MRI dynamo transition on dissipative effects, our strategy to make progress on this problem is to simplify the dynamics as much as possible and focus on a few simple dynamo cycles which encapsulate the basic physics of the dynamo. To achieve this, we enforce that the dynamics takes place in a symmetric subspace to facilitate the analysis (this does not compromise the underlying dynamical complexity, see Sect. 3 of Riols et al. 2013), and notably monitor the axisymmetric MRI-supporting field $\overline{\boldsymbol{B}}$ ( $y$-average of $\boldsymbol{B}$ ), more specifically its energetically dominant Fourier mode $\overline{\boldsymbol{B}}_{0}(z, t)=$ $\overline{\boldsymbol{B}}_{0}(t) \cos \left(k_{z_{0}} z\right)$ with $k_{z_{0}}=2 \pi / L_{z}$. We also restrict our study to the large aspect ratio configuration $\left(L_{x}, L_{y}, L_{z}\right)=(0.7,20,2)$ already employed by Herault et al. (2011) and Riols et al. (2013), and Re and $\mathrm{Rm}$ values in the range of a few hundred, which effectively guarantees that only a small number of simple nonlinear cyclic solutions are excited (see Sect. III. D of Herault et al. (2011) for a detailed explanation). Different aspect ratio configurations will be explored in a future paper.

\section{Numerical exploration of the dynamo transition}

\subsection{Dynamical lifetime of DNS in the $(R e, R m)$ plane}

As mentioned in the introduction, the numerical study of Fromang et al. (2007), for a single box size $(\pi, 2 \pi, \pi)$, in a compressible isothermal case, suggests that zero net flux MRI turbulence cannot be sustained for Pm below some critical $\mathrm{Pm}_{\mathrm{c}}$. The transitional dynamics in this box involves very intricate nonlinear interactions between many shearing waves and is therefore particularly difficult to analyse. As explained previously, we found it more convenient here to use an elongated box in which fewer shearing waves are active to address the problem of the nature of the Pm-dependence of the transition.

As a preliminary step, we first ensured that the results obtained by Fromang et al. (2007) pertain to our large aspect ratio configuration. For this purpose, we used a cartography procedure similar to that described in Riols et al. (2013). We performed a series of DNS for different Re and Rm, using the same random initial condition. The latter was generated as follows: for each field component, we generated a set of random complex Fourier 

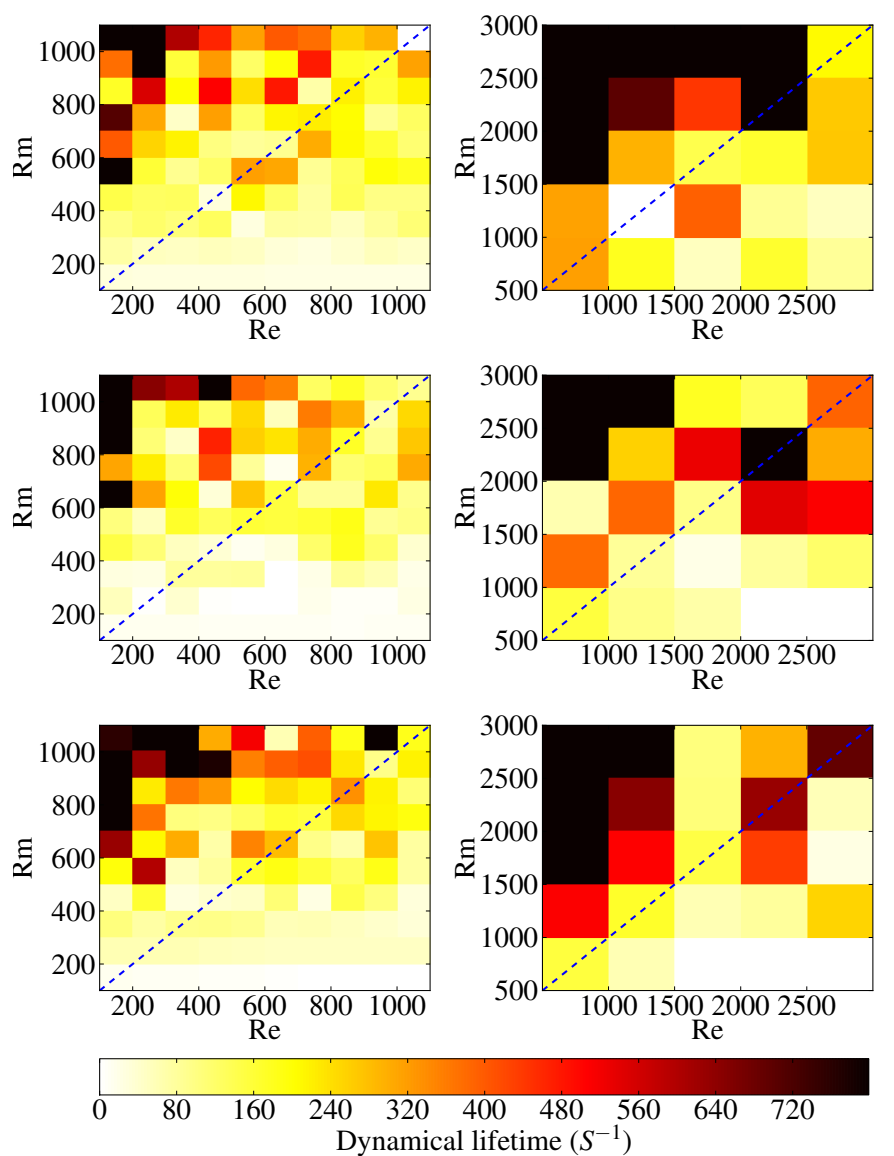

Fig. 1. Maps of the dynamical lifetime in generic DNS, as a function of $\operatorname{Re}$ and $\operatorname{Rm}\left(L_{x}=0.7, L_{y}=20, L_{z}=2\right)$. Each row is for a different random initial condition. The second and third maps (from top to bottom) use the same noise realization (see text) but different amplitudes ( $A=5$ for the second case and $A=2.5$ for the third case). The maps on the left are for simulations at mild $\mathrm{Re}$ and $\mathrm{Rm}$ that can be conducted at moderate numerical resolution $(48 \times 48 \times 72)$. The maps on the right are for higher $\mathrm{Re}$ and $\mathrm{Rm}$, requiring a higher resolution $(96 \times 96 \times 128)$. The dashed line corresponds to $\mathrm{Pm}=1$.

modes and normalized the total energy density to obtain a particular white noise incompressible "realization". For $\boldsymbol{u}$ and $\boldsymbol{B}$, a given zero net-flux initial condition is obtained by multiplying this particular noise realization by an amplitude factor $A$ (see Riols et al. (2013) for details). The typical dynamical lifetime measured in each DNS was then plotted on a two dimensional map covering the $(\mathrm{Re}, \mathrm{Rm})$ grid. To check whether the results were generic, we performed the same experiment for three different initial conditions. The first and second ones were constructed from different noise realizations but the same amplitude $A=5$. The third one used the same noise realization as the second one but with $A=2.5$ (shooting along the same direction in phase space but at different distances from the laminar state).

Figure 1 (left) shows the corresponding dynamical lifetime maps for Re and Rm between 100 and 1000, computed for a numerical resolution $48 \times 48 \times 72$. The maps on the right are for the same initial conditions, but extend to higher Re and $\mathrm{Rm}$ (from 500 to 3000 ). They required a larger numerical resolution $(128 \times 128 \times 96)$. All the DNS whose dynamical lifetime exceeds $600 S^{-1}$ are systematically on or above the Pm $\sim$ 1 line. At low Re, the dynamics seems to be sustained only for $\mathrm{Rm}$ larger than some critical $\mathrm{Rm}_{\mathrm{c}}$. At higher $\mathrm{Re}$, the transition border visually follows a $\mathrm{Pm} \simeq \mathrm{Pm}_{\mathrm{c}}$ line, with $\mathrm{Pm}_{\mathrm{c}}$ of the order of unity. This behaviour suggests that the transition border is indeed similar to that obtained by Fromang et al. (2007), and does not depend on the shearing box aspect ratio or compressibility of the fluid, at least on the qualitative level.

\subsection{Transition maps}

To justify our interest in cycles, we then attempted to check whether the conclusion of Riols et al. (2013), that chaotic dynamo action at $\mathrm{Re}=70$ results from their global bifurcations, extends to larger Re. The simplest signature of this effect is in the form of fractal-like sets of initial perturbations for which the dynamics is long-lived. To check this, we performed several series of DNS spanning a range of Rm and initial conditions, each of them generated from a unique noise realization (using the same procedure as in Sect. 3.1) and varying the perturbation amplitude $A$. We constructed maps of the dynamical lifetimes for each run as a function of $\mathrm{Rm}$ and $A$, for $\mathrm{Re}=(70,85,100,150,200,550)$.

Figure 2 shows that the boundary separating the regions in phase space where the dynamics is long-lived from those where perturbations decay rapidly has the same qualitative fractal-like structure for all Re. Long-lived simulations are characterized by recurrent dynamics reminiscent of nonlinear cycles, suggesting that the excitation of the MRI dynamo is indeed tied to that of cycles.

At the largest Re considered though, the fractal-like features appear to be smoothed out, and the correspondence between recurrent dynamics and chaotic flows less pronounced. We also note that at $\mathrm{Re}=550$, the transition border seems to be at higher $\mathrm{Rm}$ than at $\mathrm{Re}=70$, in line with the results of Fromang et al. (2007) and the results of Sect. 3.1.

Overall, the previous results suggest that the excitation of self-sustaining MHD turbulence in this problem is related to the existence of MRI dynamo cycles and their global bifurcations. This vindicates the idea that studying how the dynamics of simple nonlinear cycles is affected by changes in Re and Rm may be useful to identify the physical mechanisms responsible for the Pm-dependence of the transition.

\section{Parametric study of MRI dynamo cycles}

\subsection{Existence boundaries in the $(R e, R m)$ plane}

Motivated by the previous results, we investigated the domains of existence in parameter space of three pairs of cycles $S N_{1}$, $S N_{2}$, and $S N_{3}$ born out of saddle node bifurcations at pairspecific critical $\mathrm{Rm}_{\mathrm{c}}(\mathrm{Re}) . S N_{1}$ and $S N_{2}$ have already been documented by Riols et al. (2013) (see their Figs. 7, 8), while $S N_{3}$ was found more recently.

Continuation with respect to $\mathrm{Re}$ at fixed $\mathrm{Rm}$ of the lower and upper branches $L B_{1}$ and $U B_{1}$ of $S N_{1}$ (Fig. 3, inset) shows that they only exist in a finite range of $\mathrm{Re}$, whose extent widens as Rm increases. $S N_{2}$ and $S N_{3}$ behave similarly (not shown). The existence boundaries of all cycle pairs in the (Re, Rm) plane were constructed by combining all critical $\mathrm{Rm}_{\mathrm{c}}(\mathrm{Re})$ and $\mathrm{Re}_{\mathrm{c}}(\mathrm{Rm})$ obtained by continuation (Fig. 3). For Rm in the 300-500 range, all cycles disappear at low enough $\mathrm{Pm}$ and their $\mathrm{Rm}_{\mathrm{c}}$ increases with Re. This behaviour, reminiscent of the results of Fromang et al. (2007), is investigated below (the seemingly large differences between their transitional $\mathrm{Re}, \mathrm{Rm}$ and ours are essentially due to aspect ratio differences and do not reflect fundamental physical 

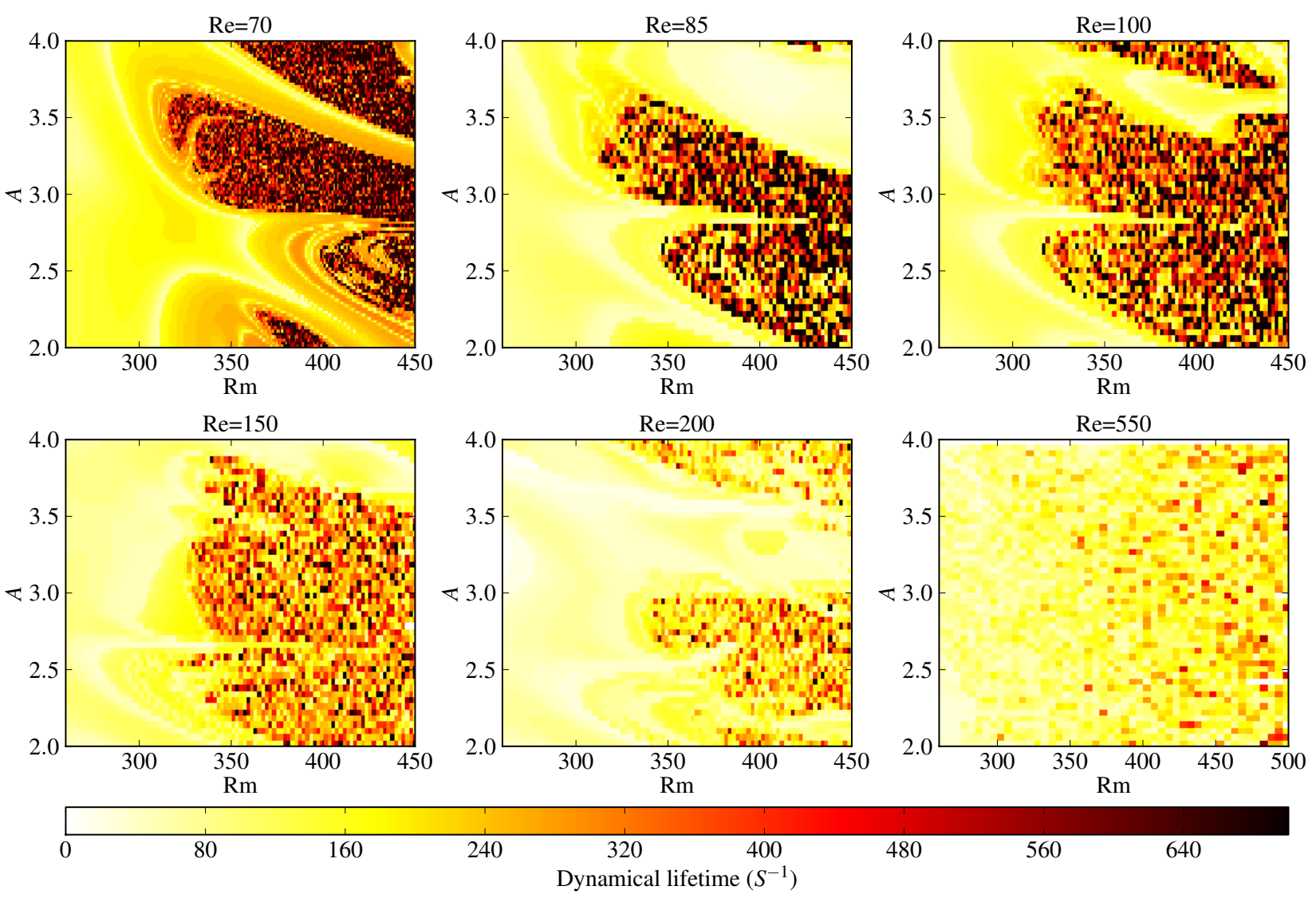

Fig. 2. Maps of dynamical lifetimes as a function of Rm and initial perturbation amplitude $A$, for a fixed noise realization (see text) and six different Re. The resolution is $(\delta A=0.04, \delta \mathrm{Rm}=2)$ except for $\operatorname{Re}=70$ where $(\delta A=0.02, \delta \mathrm{Rm}=1)$ and for $\operatorname{Re}=550$ where $\delta \mathrm{Rm}=5$.

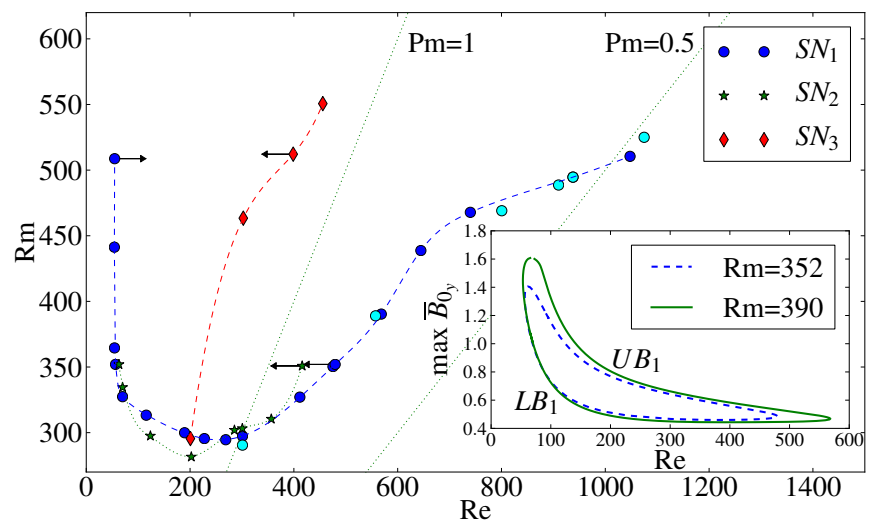

Fig. 3. Existence boundaries of three cycle pairs (dashed lines) in the $(\mathrm{Re}, \mathrm{Rm})$ plane and critical $\operatorname{Re}_{\mathrm{c}}(\mathrm{Rm}), \mathrm{Rm}_{\mathrm{c}}(\mathrm{Re})$ of saddle nodes obtained by continuation (symbols). Black arrows indicate the regions in which the cycles exist. For $S N_{1}$, dark blue/black bullets correspond to low resolution results $(24 \times 12 \times 36)$, and light blue/grey bullets are for double resolution $(48 \times 24 \times 72)$. Inset: selected continuation curves of $S N_{1}$ at fixed $\mathrm{Rm}$.

differences). For $S N_{1}$, we plotted the critical points obtained for both low resolution simulations $(24 \times 12 \times 36)$ and at higher resolution $(48 \times 24 \times 72)$. The boundary appears to be almost independent of resolution. Note that we found it very difficult to explore the strongly nonlinear regime $\mathrm{Rm}>500$ at the maximum resolution considered. Tentative results at this resolution (not shown) suggest that $S N_{1}$ may have an upper boundary in $\mathrm{Rm}$ in this regime.

\subsection{Magnetic energy budgets of MRI dynamo cycles}

As the MRI dynamo rests on the sustainment of the axisymmetric MRI-supporting field $\overline{\boldsymbol{B}}_{0}$ against dissipative processes through nonlinear interactions of non-axisymmetric modes, analysing the magnetic energy budget of dynamo cycles, most importantly transitional lower branch saddles such as $L B_{1}$, may give useful physical insights into the $\mathrm{Pm} \leq 1$ regime. To do this, we write

$$
\boldsymbol{B}=\overline{\boldsymbol{B}}_{0}+\boldsymbol{b}_{1}+\sum_{j \geqslant 2} \boldsymbol{b}_{j} \quad \text { and } \quad \boldsymbol{u}=\boldsymbol{u}_{1}+\sum_{j \geqslant 2} \boldsymbol{u}_{j},
$$

where $\boldsymbol{u}_{1}$ and $\boldsymbol{b}_{1}$ stand for non-axisymmetric "MRI wave" perturbations supported by shearing waves with wavenumbers $\left|k_{y}\right|=$ $k_{y_{0}}=2 \pi / L_{y}, k_{x}(t)=S k_{y} t,\left|k_{z}\right|=k_{z_{0}}$ for $\boldsymbol{u}_{1}$ and $k_{z}=0$ for $\boldsymbol{b}_{1}$.

The sum over $j \geq 2$ stands for all other (smaller-scale) fluctuations.

We first integrate the energy equation for $\overline{\boldsymbol{B}}_{0}(z, t)$ over the volume and half cycle period $T / 2=S^{-1} L_{y} / L_{x}$. Denoting this operation by \langle\rangle and taking into account that cyclic magnetic reversals change $\overline{\boldsymbol{B}}_{0}$ into $-\overline{\boldsymbol{B}}_{0}$, we obtain the energy budget 
A. Riols et al.: Dissipative effects on the sustainment of a magnetorotational dynamo in Keplerian shear flow
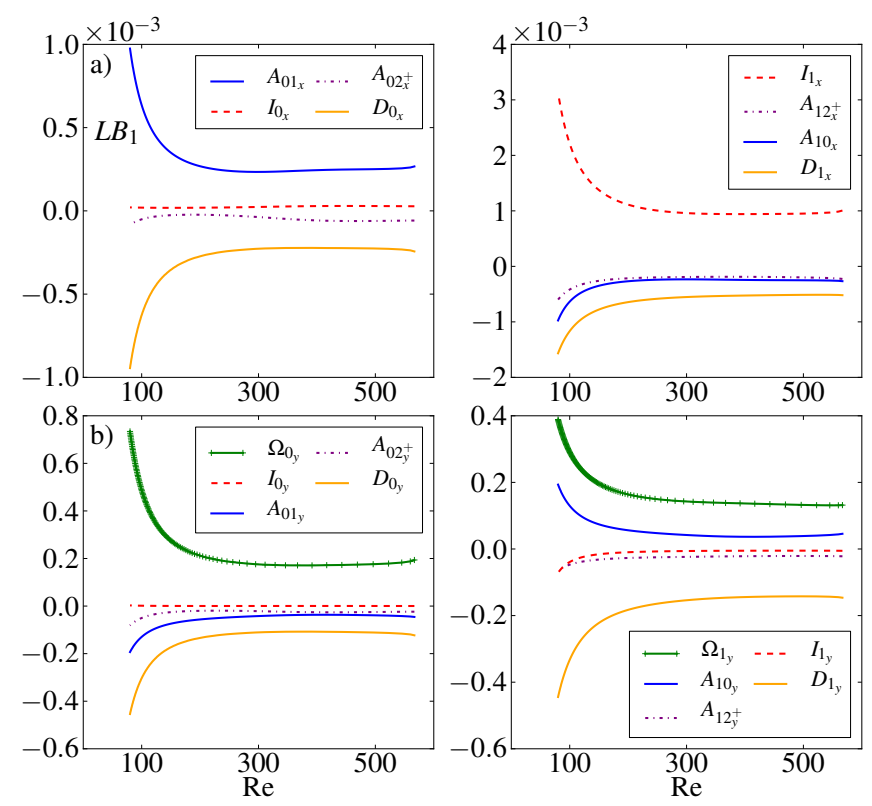

Fig. 4. Magnetic energy budgets as a function of Re: a) $x$ projection of the budgets for $\overline{\boldsymbol{B}}_{0}($ left $)$ and $\boldsymbol{b}_{1}($ right $)$ for $L B_{1}$ at $\mathrm{Rm}=390, \mathbf{b}$ ) corresponding $y$ projection.

for $\overline{\boldsymbol{B}}_{0}$,

$\boldsymbol{\Omega}_{0}+\boldsymbol{I}_{0}+\boldsymbol{A}_{0}+\boldsymbol{D}_{0}=\mathbf{0}, \quad$ where

$\boldsymbol{\Omega}_{0}=-S\left\langle\bar{B}_{0_{y}} \bar{B}_{0_{x}}\right\rangle \boldsymbol{e}_{y}, \quad \boldsymbol{I}_{0}=\left\langle\overline{\boldsymbol{B}}_{0} \circ \overline{\boldsymbol{B} \cdot \boldsymbol{\nabla} \boldsymbol{u}}\right\rangle$,

$\boldsymbol{D}_{0}=-\eta k_{z_{0}}^{2}\left\langle\overline{\boldsymbol{B}}_{0} \circ \overline{\boldsymbol{B}}_{0}\right\rangle, \quad \boldsymbol{A}_{0}=\boldsymbol{A}_{01}+\boldsymbol{A}_{02^{+}}$,

$\boldsymbol{A}_{01}=-\left\langle\overline{\boldsymbol{B}}_{0} \circ \overline{\boldsymbol{u}_{1} \cdot \boldsymbol{\nabla} \boldsymbol{b}_{1}}\right\rangle, \quad \boldsymbol{A}_{02^{+}}=-\left\langle\overline{\boldsymbol{B}}_{0} \circ \overline{\boldsymbol{u} \cdot \boldsymbol{\nabla} \boldsymbol{B}}\right\rangle-\boldsymbol{A}_{01}$,

and $\circ$ is the Hadamard (entrywise) product; $\boldsymbol{\Omega}_{0}$ is the energy provided by the linear stretching of $\bar{B}_{0_{x}}$ by the shear ( $\Omega$ effect) and $\boldsymbol{I}_{0}$ is a nonlinear induction term; $\boldsymbol{A}_{0}$ is the magnetic energy exchanged with other modes through nonlinear advection and $\boldsymbol{D}_{0}$ is the ohmic dissipation; $\boldsymbol{A}_{01}$ is the energy exchanged with the MRI-unstable waves and $\boldsymbol{A}_{02^{+}}$is the energy exchanged with all $j \geq 2$ modes. Figure 4a,b (left) display the $x$ and $y$ projections of Eq. (5) for $L B_{1}$ as a function of Re, at fixed $\mathrm{Rm}\left(U B_{1}\right.$ is more energetic but behaves similarly). The MRI-supporting azimuthal field $\bar{B}_{0_{y}}$ loses energy through laminar dissipation $D_{0_{y}}$, but also through a nonlinear advective transfer to other modes $A_{0_{y}}<0$, which therefore acts as a weakly nonlinear ("turbulent") diffusion. The $\Omega$ effect is the only net source term for $\bar{B}_{0_{y}}$, therefore the sustainment of $\bar{B}_{0_{x}}$ is critical for the dynamo. Figure $4 \mathrm{a}$ (left) shows that $\bar{B}_{0_{x}}$ gains energy from the nonlinear term $A_{01_{x}}>0$, which is the product of the MRI correlation of $\boldsymbol{u}_{1}$ and $\boldsymbol{b}_{1}$, and loses energy via $D_{0_{x}}$ and $A_{02_{x}^{+}}<0$, so that $A_{01_{x}} \simeq\left|D_{0_{x}}+A_{02_{x}^{+}}\right|$. $A_{02_{x}^{+}}$transfers energy to smaller scales (where it is dissipated), and can again be interpreted as a nonlinear diffusion of $\bar{B}_{0_{x}}$.

To understand how energy is injected into the MRI wave and transferred to $\overline{\boldsymbol{B}}_{0}$, we now consider the energy budget for $\boldsymbol{b}_{1}$,

$\mathbf{\Omega}_{1}+\boldsymbol{I}_{1}+\boldsymbol{A}_{1}+\boldsymbol{D}_{1}=\left\langle\boldsymbol{b}_{1} \circ\left(\partial \boldsymbol{b}_{1} / \partial t\right)\right\rangle \simeq \mathbf{0}$, where

$\boldsymbol{\Omega}_{1}=-S\left\langle\bar{b}_{1_{y}} \bar{b}_{1_{x}}\right\rangle \boldsymbol{e}_{y}, \quad \boldsymbol{I}_{1}=\boldsymbol{I}_{1 L}+\boldsymbol{I}_{1 N L}$,

$\boldsymbol{D}_{1}=-\eta\left\langle\left(k_{x}(t)^{2}+k_{y_{0}}^{2}\right) \boldsymbol{b}_{1} \circ \boldsymbol{b}_{1}\right\rangle, \quad \boldsymbol{A}_{1}=\boldsymbol{A}_{10}+\boldsymbol{A}_{12^{+}}$,

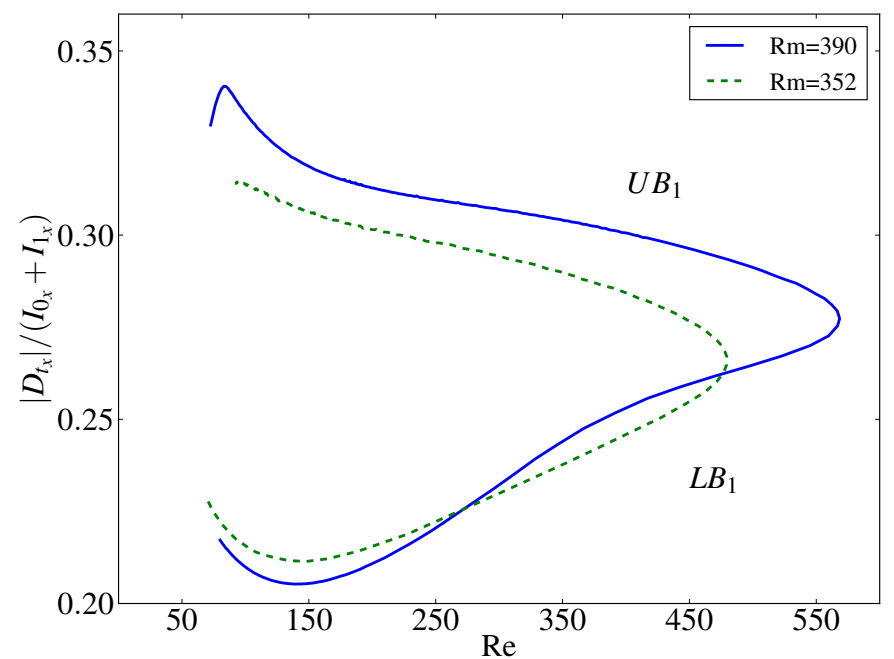

Fig. 5. Ratio between nonlinear dissipation $D_{t_{x}}$ and injected energy $I_{0_{x}}+$ $I_{1}$ for $L B_{1}$ and $U B_{1}$ and two different $\mathrm{Rm}$.

$\boldsymbol{I}_{1 L}=\left\langle\boldsymbol{b}_{1} \circ\left(\overline{\boldsymbol{B}}_{0} \cdot \boldsymbol{\nabla} \boldsymbol{u}_{1}\right)\right\rangle$ and $\boldsymbol{I}_{1 N L}$ is a nonlinear induction term; $\boldsymbol{A}_{10}=-\boldsymbol{A}_{01}=-\left\langle\boldsymbol{b}_{1} \circ\left(\boldsymbol{u}_{1} \cdot \boldsymbol{\nabla} \overline{\boldsymbol{B}}_{0}\right)\right\rangle$ is the energy exchanged through advection between the MRI wave and $\overline{\boldsymbol{B}}_{0}$, and $\boldsymbol{A}_{12^{+}}$accounts for a similar exchange with smaller scales (Eq. (9) is almost zero because the wave carries a negligible amount of energy at both $t=0$ and $t=T / 2$ ). Figure 4a,b (right) shows that the $x$ and $y$ components of the MRI perturbation $\boldsymbol{b}_{1}$ are fed by induction (respectively by $I_{1_{x}}$ and $\Omega_{1_{y}}$, with $I_{1 N L_{x}} \ll I_{1 L_{x}}$ ). As expected, some of the energy injected via the MRI is transferred back nonlinearly to $\bar{B}_{0_{x}}$ through $A_{10_{x}}=-A_{01_{x}}$, and some of it is lost through laminar dissipation $D_{1_{x}}$. The rest $A_{12_{x}^{+}}<0$ is transferred to smaller scales and can be regarded as a nonlinear diffusion of MRI-unstable perturbations. Using a similar analysis, we checked that $j \geq 2$ perturbations are mostly excited via nonlinear interactions, and not by the MRI, for all cases studied here. Summing Eqs. (5) and (9) to eliminate $A_{01_{x}}$, we obtain

$I_{0_{x}}+I_{1_{x}} \simeq\left|D_{0_{x}}+D_{1_{x}}\right|+\left|D_{t_{x}}\right|$,

which translates that the energy injected via the MRI as $I_{1_{x}}$ must balance the total of "laminar" ohmic dissipation $\left|D_{0_{x}}+D_{1_{x}}\right|$ and nonlinear dissipation $\left|D_{t_{x}}\right| \equiv\left|A_{12_{x}^{+}}+A_{02_{x}^{+}}\right|$for the dynamo to be sustained $\left(I_{0_{x}} \ll I_{1_{x}}\right.$ in all cases studied here).

To understand how Eq. (12) is satisfied in different regimes, we show in Fig. 5 the ratio $\left|D_{t_{x}}\right| /\left(I_{0_{x}}+I_{1_{x}}\right)$ for $L B_{1}$ and $U B_{1}$ as a function of $\mathrm{Re}$, for two values of $\mathrm{Rm}$. This ratio is always significantly larger for $U B_{1}$ than for $L B_{1}$, which is consistent with the standard picture of upper branches being more nonlinear than lower branches. The amplitude of $\overline{\boldsymbol{B}}_{0}$ is larger on $U B_{1}$ (Fig. 3, inset), which results in a stronger MRI driving (the MRI is always in the regime $k_{y_{0}} \bar{B}_{0_{y}}<\Omega$ here, so the stronger the field, the larger the growth rate). The most important observation, however, is the significant increase of $\left|D_{t_{x}}\right| /\left(I_{0_{x}}+I_{1_{x}}\right)$ with Re for the saddle solution $L B_{1}$, which shows that a larger fraction of the energy injected in $\overline{\boldsymbol{B}}_{0}$ and $\boldsymbol{b}_{1}$ is lost through nonlinear dissipation $D_{t_{x}}=A_{12_{x}^{+}}+A_{02_{x}^{+}}$as Re increases. Our interpretation is that this relative enhancement of "turbulent" magnetic diffusion is tied to the facilitated excitation of velocity fluctuations at large Re. 

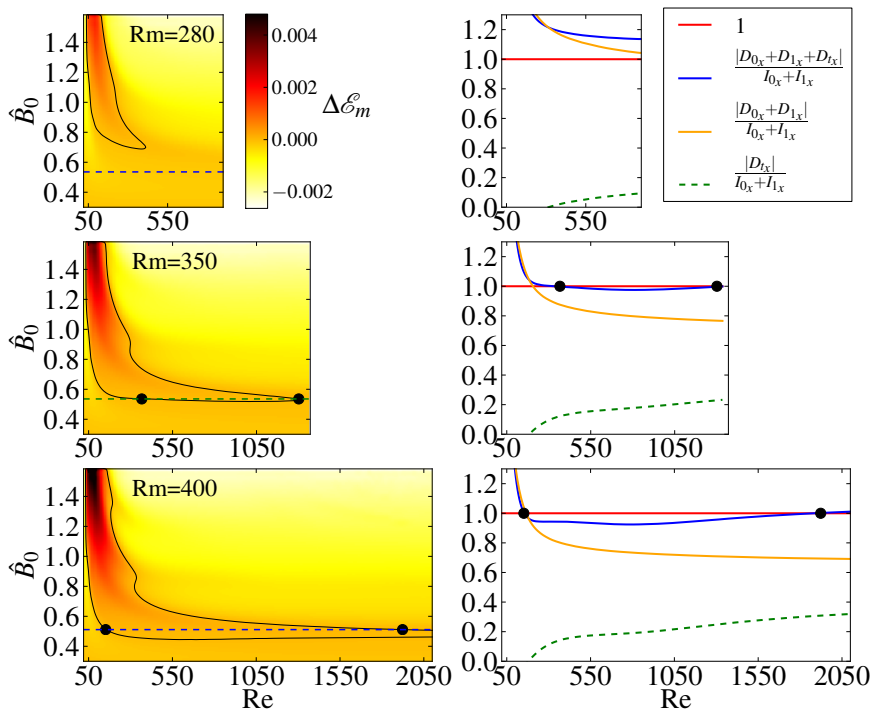

Fig. 6. Energy budget of simulations integrated over $S t=L_{y} / L_{x}$ for three values of Rm. Left: net energy gain $\Delta \mathcal{E}_{m}$ as a function of Re and $\hat{B}_{0} . \Delta \mathcal{E}_{m}=0$ isolines are shown in black, bullets mark the points $\Delta \mathcal{E}_{m}=$ 0 for $\hat{B}_{0} \simeq 0.52$. Right: normalized injection and dissipation terms in Eq. (12), as a function of Re, for the same $\hat{B}_{0}$.

\section{Disappearance of the dynamo}

The previous results provide qualitative clues to understand the conditions of excitation of the dynamo. We note that the amplitude of $\overline{\boldsymbol{B}}_{0}$ on $L B_{1}$ seems to asymptote to a constant at large Re and fixed Rm (Fig. 3 (inset)), and therefore so should the MRI growth rate for this branch. This suggests that the MRI may not be able to sustain $\overline{\boldsymbol{B}}_{0}, \boldsymbol{b}_{1}$ and therefore the dynamo against the total effective magnetic diffusion beyond some critical $\mathrm{Re}$, as observed in Figs. 3 and 5. To investigate more precisely how the energy balance of Eq. (12) may be broken, we prepared a family of initial conditions resembling $L B_{1}$ at $t=0$, consisting of an axisymmetric field $\overline{\boldsymbol{B}}_{0}=\hat{\boldsymbol{B}}_{0}\left(0.04 \boldsymbol{e}_{x}+\boldsymbol{e}_{y}\right)$ parametrized by $\hat{B}_{0}$, plus non-axisymmetric perturbations in the form of a given packet of shearing waves $\left(\left|k_{y}\right|=k_{y_{0}}, k_{x}(t=0)=0\right.$ and multiple $\left.k_{z}\right)$ with weak but random amplitudes $\left(\bar{B}_{0_{x}} / \bar{B}_{0_{y}}=0.04\right.$ is representative of $L B_{1}$ and ensures that $\Omega_{0_{y}}$ is of the order of $\left.D_{0_{y}}\right)$. This set of initial conditions was then integrated by DNS during half a cycle period typical of a reversal of $\overline{\boldsymbol{B}}_{0}$, for a range of Re and $\hat{B}_{0}$. The results were used to construct a map of the net energy $\Delta \mathcal{E}_{m}=I_{0_{x}}+I_{1_{x}}-\left|D_{0_{x}}+D_{1_{x}}+D_{t_{x}}\right|$ gained or lost by the active magnetic modes during the reversal, as a function of $\operatorname{Re}$ and $\hat{B}_{0}$, for several Rm (Fig. 6 (left)). The isolines $\Delta \mathcal{E}_{m}=0$ are reminiscent of the continuation curves of $S N_{1}$ (Fig. 3 (inset)). The range of $\mathrm{Re}$ in which the system gains more energy from the MRI than it dissipates $\left(\Delta \mathcal{E}_{m}>0\right)$ widens significantly at larger $\mathrm{Rm}$.

Figure 6 (right) shows plots of the different terms in Eq. (12) normalized by $\left(I_{0_{x}}+I_{1_{x}}\right)$ as a function of $\mathrm{Re}$, for $\hat{B}_{0} \simeq 0.52$. At $\mathrm{Rm}=280$, the system loses energy for all Re after a reversal. At $\mathrm{Rm}=350$, there is a range of $\mathrm{Re}$ in which more energy is pumped in by the MRI than dissipated. This range widens at even larger $\mathrm{Rm}=400$. The transition from a sustained to a decaying regime at large $\mathrm{Re}$ occurs because $\left|D_{0_{x}}+D_{1_{x}}\right| /\left(I_{0_{x}}+I_{1_{x}}\right)$ tends to a constant at large Re, while $\left|D_{t_{x}}\right| /\left(I_{0_{x}}+I_{1_{x}}\right)$ increases slowly. The reason why the dynamo can be sustained at larger Re as $\mathrm{Rm}$ increases is that the MRI growth rate is not asymptotic in
$\mathrm{Rm}$ in the transitional 300-500 $\mathrm{Rm}$ range. Laminar dissipation is reduced relative to energy injection as $\mathrm{Rm}$ increases, which partially offsets the increase in "turbulent" diffusion at large Re. Equivalently, we may conclude that this increase at large Re requires to go to larger $\mathrm{Rm}$ to recover the dynamo, as observed in Figs. 2 and 3 and reported by Fromang et al. (2007).

\section{Discussion}

Why is the MRI dynamo in Keplerian flow harder to excite at low Pm? Using a simple numerical setup, we have found that weakly nonlinear "turbulent" diffusion (in a qualitative, not strictly mean-field theoretical sense) of large-scale magnetic modes makes it increasingly difficult to sustain the dynamo at moderate $\mathrm{Rm}$ as $\mathrm{Re}$ increases. The significant advective transfers of magnetic energy to small scales reported by Fromang et al. (2007) in smaller aspect ratio simulations at large Re corroborate this conclusion. A subtle point is that the velocity fluctuations behind turbulent magnetic diffusion in this subcritical problem are not externally prescribed but are indirectly transiently excited by the MRI.

Turbulent diffusion has also been measured in turbulent flows of low Pm liquid metals (Frick et al. 2010; Rahbarnia et al. 2012), in which it is strongly suspected of raising (kinematic) dynamo thresholds (Miralles et al. 2013). Given the very generic nature of this effect, we therefore argue that it could be an important determinant of MRI dynamo excitation in low Pm rotating shear flows, such as occur in parts of some accretion disks (some of which also have low Rm). Besides, the fact that it also affects MRI-active modes suggests that it may be linked to the drop in angular momentum transport reported in net-flux (imposed field) MRI simulations at (moderately) low Pm.

More work is clearly required to connect these results to the full diversity of simulated and astrophysical regimes, most importantly the numerically challenging limit $\mathrm{Re} \gg \mathrm{Rm} \gg 1$, and to study to which extent the conclusions pertain to numerical configurations with different aspect ratio. A similar preliminary study in smaller aspect ratio boxes suggests that the same qualitative conclusions apply in this case. These results will be presented in a future paper. Other nonlinear effects, some of which may qualitatively relate to the mean-field theoretical $\alpha$ effect with vertical stratification, are also probably very important in the disk dynamo problem (Brandenburg et al. 1995; Gressel 2010; Davis et al. 2010; Käpylä \& Korpi 2011; Oishi \& Mac Low 2011; Simon et al. 2011; Blackman 2012) and will be worthwhile of investigation along the same lines.

Acknowledgements. This research was supported by the University Paul Sabatier of Toulouse under an AO3 grant, by the Midi-Pyrénées region and by the French National Program for Stellar Physics (PNPS). Numerical calculations were carried out on the CALMIP platform (CICT, University of Toulouse), whose assistance is gratefully acknowledged.

\section{References}

Balbus, S. A., \& Hawley, J. F. 1991, ApJ, 376, 214

Balbus, S. A., \& Hawley, J. F. 1998, Rev. Mod. Phys., 70, 1

Balbus, S. A., \& Henri, P. 2008, ApJ, 674, 408

Blackman, E. G. 2012, Phys. Scr., 86, 058202

Bodo, G., Cattaneo, F., Ferrari, A., Mignone, A., \& Rossi, P. 2011, ApJ, 739, 82 Brandenburg, A., Nordlund, A., Stein, R. F., \& Torkelsson, U. 1995, ApJ, 446, 741

Chandrasekhar, S. 1960, Proc. Natl. Acad. Sci., 46, 253 
A. Riols et al.: Dissipative effects on the sustainment of a magnetorotational dynamo in Keplerian shear flow

Davis, S. W., Stone, J. M., \& Pessah, M. E. 2010, ApJ, 713, 52

Donati, J.-F., Paletou, F., Bouvier, J., \& Ferreira, J. 2005, Nature, 438, 466 Frick, P. A., Noskov, V. I., Denisov, S. A., \& Stepanov, V. A. 2010, Phys. Rev. Lett., 105, 184502

Fromang, S., Papaloizou, J., Lesur, G., \& Heinemann, T. 2007, A\&A, 476, 1123

Goldreich, P., \& Lynden-Bell, D. 1965, MNRAS, 130, 125

Gressel, O. 2010, MNRAS, 405, 41

Hawley, J. F., Gammie, C. F., \& Balbus, S. A. 1995, ApJ, 440, 742

Hawley, J. F., Gammie, C. F., \& Balbus, S. A. 1996, ApJ, 464, 690

Herault, J., Rincon, F., Cossu, C., et al. 2011, Phys. Rev. E, 84, 036321

Käpylä, P. J., \& Korpi, M. J. 2011, MNRAS, 413, 901
Lesur, G., \& Longaretti, P.Y. 2007, MNRAS, 378, 1471

Lesur, G., \& Ogilvie, G. I. 2008, A\&A, 488, 451

Miralles, S., Bonnefoy, N., Bourgoin, M., et al. 2013, Phys. Rev. E, 88, 013002

Oishi, J. S., \& Mac Low, M.-M. 2011, ApJ, 740, 18

Rahbarnia, K., Brown, B. P., Clark, M. M., et al. 2012, ApJ, 759, 80

Rincon, F., Ogilvie, G. I., \& Proctor, M. R. E. 2007, Phys. Rev. Lett., 98, 254502

Rincon, F., Ogilvie, G. I., Proctor, M. R. E., \& Cossu, C. 2008, Astron. Nachr., 329, 750

Riols, A., Rincon, F., Cossu, C., et al. 2013, J. Fluid Mech., 731,

Simon, J. B., Hawley, J. F., \& Beckwith, K. 2011, ApJ, 730, 94

Velikhov, E. P. 1959, Sov. Phys. JETP, 36, 1398 